\title{
High rate of Section 4 admissions: clinical implications and possible explanation
}

\author{
Basant K. PURI, Honorary Registrar in Psychiatry, Addenbrookes Hospital, \\ Cambridge; and Donald F. Bermingham, Consultant Psychiatrist, Hinchingbrooke \\ Hospital, Huntingdon, and Fulbourn Hospital, Cambridge
}

The Mental Health Commissioners recommend that whenever possible two doctors should be involved in the decision to admit a patient to hospital under the Mental Health Act (1983). That is, Sections 2 and 3 of the Act should always be used in preference to Section 4 , where only one doctor is required. It further recommends that the use of Section 4 should be confined to emergencies when it is only possible to secure the attendance of one doctor.

It came to our attention that the Commissioners had noted a high level of admission under Section 4 to Fulbourn Hospital, Cambridge. The national and local Cambridge figures for 1986 reveal that while Section 4 admissions comprised $18 \%$ of all admissions under the Mental Health Act $(2,738$ out of 15,250 ) nationally, in Cambridge they made up $30 \%$ of all such admissions ( 33 out of 112 ).

While it is clearly good clinical practice to involve two doctors in the important decision of admission under a Section, we wondered whether being admitted under Section 4 was related to any difference to the patient with respect to the diagnosis, management in hospital, and follow-up. A pilot study was therefore carried out to compare the hospital careers of patients admitted in Cambridge during 1986 under Section 2, Section 4, and informally (the control group).

\section{The study}

The first 50 admissions under Section 2 or Section 4 to Fulbourn Hospital in 1986 were studied. By coincidence there were 25 in each group, and these were matched with a random sample of 25 informal admissions to the same wards in 1986. The parameters studied included age, sex, number and status of previous admissions, case note diagnosis, length of the current admission, type of medication prescribed, and follow-up arrangements. In addition, the 1986 national figures for admissions under the various Sections of the Mental Health Act were compared with the corresponding figures for Cambridge.

\section{Findings}

When compared to the control group, admissions to Fulbourn Hospital under Sections 2 and 4 were found to be similar in terms of age (mean $\mathbf{4 4}$ and $\mathbf{4 5}$ years, respectively), numbers of previous admissions $(74,73)$, length of stay in hospital, and type of treatment prescribed (neuroleptics 21, 28; benzodiazepines 4, 5; lithium 4, 5; antidepressants 3, 5; ECT 1, 3; nil 1,1$)$. Indeed the similarities in the length of stay in hospital (for example, the numbers staying for less than six months were 11 for Section 2 admissions, and 12 for Section 4) were significant when compared with informal admissions $(P<0.01)$. Furthermore, while admissions under Sections 2 and 4 were more likely to be prescribed neuroleptics and lithium (see above figures), the informal admissions were found to be more likely to be prescribed antidepressants (10), benzodiazepines (12), or nothing at all (4). (Note that for the medication data, $n=23$ for Sections 2 and $4 ; n=24$ for the control group).

There were a number of parameters on which Section 4 admissions were found to be similar to the informal admissions. In the figures which follow, the Section 4 figure is given first, followed by the corresponding informal admissions figure, with the Section 2 figure being given in parentheses for comparison. Ratio of males to females - 1:1.8, 1:1.8 (1:0.8). Diagnosis of mood disorder $-76 \%, 56 \%$ (47\%); schizophrenia - 19\%, $24 \%$ (32\%); organic disorder - $5 \%$, $8 \%(11 \%)$; neurosis - $0 \%$, $4 \%$, $(0 \%)$; drug addiction $-0 \%, 8 \%,(0 \%)$; personality disorder $-0 \%, 0 \%$ (11\%). Out-patient follow-up - 58\%, 72\% (35\%).

When examining the admission figures under the various Sections of the Mental Health Act (1983) it was found that in $198610 \%$ of all Section admissions nationally were under Section 136, while the corresponding figure for Cambridge was only $1 \%$. It has already been mentioned above that in the same year Section 4 admissions comprised $18 \%$ of all Section admissions nationally, and $30 \%$ of Section admissions to Cambridge. Thus, if Sections 4 and 136 are accepted as 'emergency Sections', then these figures indicate that the percentage of cases dealt with as an emergency are similar ( $28 \%$ nationally 
and $31 \%$ in Cambridge); however, there is a marked difference as to whether Section 4 or Section 136 is used.

\section{Comment}

While it is unwise to draw firm conclusions from the small numbers in this study, nevertheless there are indications that a patient admitted under Section 4 would appear to have a 'patient profile' in between that of a Section 2 patient and an informal patient, and possibly closer to that of a Section 2 patient. This may reflect the intermediary status of this group of patients. We feel it is more likely to reflect the possibility that some of these patients closely fit the Section 2 profile, while another group closely fit that of the control. The latter group might, then, possibly have been more appropriately admitted informally or not at all. The hypothesis we have put forward finds some further, albeit indirect, support in the conversion records of the Section 4 patients. Half of them were converted within 72 hours of admission to informal status, while the other half were converted during the same period into Section 2.

The reason why there is such a high Section 4 rate in Cambridge will now be considered. There are, under the Mental Health Act (1983), two Sections which have as their primary function the response to a psychiatric emergency in the community. The first is Section 4, which allows for the opinion of only one doctor on the grounds that there would be an unreasonable delay if the opinion of two doctors were to be required. The other is Section 136, which allows a police officer to remove to a place of safety a person found in a public place who appears, to that police officer, to be suffering from a mental disorder and to be in immediate need of care and attention. It is clear from our results that Cambridge has approximately the same percentage of emergency work as the national average. The difference is that in Cambridge it is dealt with by involving Section 4, whereas nationally the police are involved in a high percentage of cases. It is enticing to speculate that these contrasting figures may be causally related. This would raise the question as to why this should be the case. One of the authors (DB) has been involved in looking at areas where there is a high rate of Section 136 admissions (Fahy et al, 1987) and it has been noted that there are a variety of explanations. These include large urban populations with large ethnic minorities and lack of provision of approved social workers. (An approved social worker is essential for Sections 4 and 2 but not for Section 136).

Webster et al (1987), when reviewing the effect of the Mental Health Act (1983), remarked that there had been a reduction in the use of emergency orders and that patients were now being admitted either informally or on alternative orders. Their paper noted that under the previous Mental Health Act (1959), Section 29 had comprised $3.2 \%$ of Sections and under the new Act the equivalent Section 4 only comprised $1.6 \%$. However, it is interesting to note that during the same period they found a fivefold increase in Section 136 admissions, admittedly from a very small baseline. The authors remarked that they were unable to explain this increase.

Fulbourn Hospital does not serve a particularly dense urban population. There are no major ethnic minorities and there is 24 hour social worker support. However, Cambridge has yet to respond fully to the Commissioners' recommendations that two doctors be involved in every Section. The results of the first part of this study suggest that if these recommendations were implemented it might prove possible to reduce the overall Section rate still further, possibly by as much as $15 \%$.

Finally, it should be noted that although this paper has discussed Sections 2, 4 and 136 as if they were all Sections for Compulsory Admission under the Act, Section 136 is in fact not an admission Section, but is intended to be used for assessment in order to decide whether admission is needed, either informally or under Section 2 or 3. However, both this study and previous ones (Fahy et al, 1987) indicate that in practice Section 136 often results in patients being brought to a psychiatric hospital to be assessed, and in these instances the distinction between assessment and compulsory admission to hospital is blurred.

\section{References}

Fahy, T. A., Bermingham, D. \& Dunn, J. (1987) Police admissions to psychiatric hospitals: a challenge to community psychiatry? Medicine, Science and the Law, 4, 263-268.

Webster, L., DeAn, C. \& Kessel, N. (1987) Effects of the 1983 Mental Health Act on the management of psychiatric patients. British Medical Journal, 295, 1529-1532. 